Esta obra está bajo una Licencia Creative Commons Atribución - No Comercial - Sin Derivar 4.0 Internacional

Memoria mediática: la nostalgia en la cultura pop

José Antonio Hernández-Gutiérrez

Question, Vol. 1, N. 62, e152, abril-junio 2019

ISSN 1669-6581 | https://doi.org/10.24215/16696581e152

http://perio.unlp.edu.ar/ojs/index.php/question

FPyCS | Universidad Nacional de La Plata

La Plata | Buenos Aires | Argentina

\title{
Memoria mediática: la nostalgia en la cultura pop
}

\author{
Mediatic memory: nostalgia in pop culture
}

\author{
José Antonio Hernández-Gutiérrez dr.jose.hernandez@itesm.mx \\ http://orcid.org/0000-0001-5013-0741 \\ Escuela de Humanidades y Educación; Tecnológico de Monterrey (México)
}

Resumen

Cuando se habla de nostalgia se alude la parte emotiva y sensible de los sujetos, pero necesariamente también se le asocia con los recuerdos, con la reminiscencia, es decir, con la memoria. Sin embargo, no se trata sólo de las evocaciones que tenemos de forma personal o individual, sino también de los recuerdos colectivos y las experiencias que se comparten grupalmente en torno a ellos. En este artículo se hace una revisión de algunas aproximaciones teóricas que consideran que la memoria colectiva está cumpliendo un papel elemental para la 
comprensión de las identidades que se están re-construyendo en el presente. De hecho, algunos autores afirman que las sociedades valoran más la memoria cuando las identidades entran en crisis. Asimismo, se expone que una de las consecuencias de la masificación de la cultura popular, a través de los medios de comunicación y la industria del entretenimiento, fue la gestación de una memoria colectiva de tipo mediático cuyo rescate estético por parte de los grupos generacionales -independientemente de su reexplotación comercial- está sirviendo como referente socio-cultural para reconocer su pasado, crear conjuntamente su propia versión de la historia y reflexionar acerca del presente.

Palabras clave: Memoria mediática; memoria colectiva; nostalgia cultural; cultura pop.

\section{Abstract}

When we speak of nostalgia, we allude the emotive and sensitive part of the subjects, but necessarily it is also associated with memories, reminiscence, and memory. However, it is not only about the evocations we have personal or individually but also about the collective memories and experiences that groups share. In this article, we review several the oretical approaches that consider collective memory is fulfillingan elementary role fo runderstanding the identities reconstructed in the present. Some authors affirm that societies value memory more when identitiesenterinto crisis. Like wise, it is exposed that one of the consequences of the massification of popular culture, through the media and the entertainment industry, was the gestation of a collective memory of media type. Its a esthetic rescue, by generational groups, regardles $s$ of its commercial re-exploitation- is also serving as a socio-cultural referent to recognize the past of the subjects, create their own version of history and reflect on the present.

Keywords: Mediatic memory; colective memory; cultura nostalgia; pop culture.

El sociólogo clásico Emile Durkheim fue uno de los primeros científicos sociales en afirmar que toda memoria es social y que la misma sociedad determina qué es importante recordar. Asimismo, enfatizó la importancia de mantener viva la relación de las sociedades con su pasado, pues -según él- cada comunidad requiere un sentido de continuidad y unidad con el pasado por medio de los recuerdos perdurables. En Las formas elementales de la vida religiosa (2003), el teórico francés comentó que el pasado se reproduce y se renueva a través de la 
periodización de los ritos conmemorativos. Esto es, los pueblos perpetúan sus tradiciones a través de la transmisión generacional de los objetos y eventos sagrados.

Sumándose a esta postura, uno de los alumnos y críticos de Durkheim, Maurice Halbwachs, propuso una teoría de la memoria colectiva. Inclusive, su maestro previamente había destacado que sería importante explorar cómo se desarrolla socialmente la construcción de la conciencia y la memoria, así como la psicología ya se había encargado de hacerlo desde lo individual. Pese a que, desde la visión psicológica, el individuo es el sujeto que recuerda, desde esta perspectiva sociológica la memoria es un hecho social, dado que siempre está inserta en marcos sociales (Halbwachs, 2004) y es acompañado por factores compartidos como el lenguaje, los valores, los rituales y las prácticas conmemorativas.

Sobre este asunto, María del Carmen de la Peza, investigadora y especialista mexicana en medios masivos, en su estudio sobre el papel del bolero en la educación sentimental en el México contemporáneo (2001), afirma que la memoria individual y la colectiva siempre están en contacto y se alimentan mutuamente, pues cada individuo es apto para recordar y recrear el pasado recurriendo a un determinado contexto grupal; asimismo nuestros sentimientos y pensamientos más personales tienen su origen en medios y condiciones sociales. Por lo tanto, no se puede asegurar que todo lo que está registrado en la memoria individual esté desligado de las relaciones con otras personas, quienes seguramente tienen recuerdos similares respecto a las experiencias vividas en un pasado intersubjetivo. La memoria es una importante fuente para la construcción de las identidades grupales, pues no podemos desligarnos de todo lo que hemos adquirido en las experiencias anteriores. De ahí que la nostalgia cultural también muestre indicios de ser un fenómeno social vinculado a un pasado y una memoria comunes a los miembros de las generaciones.

Según Halbwachs (1992), no únicamente los sucesos históricos son significativos para los recuerdos de las personas, sino que también influye todo lo que se guarda en la memoria colectiva. Ésta, según él, es una forma de reconstruir socialmente el pasado -no de preservarla- basada en los intereses y necesidades que en la actualidad forman parte del entorno grupal de las familias, las comunidades, las naciones y las generaciones. Así, nuestros intereses y necesidades, tanto personales como colectivos, son factores que nos permiten tener una perspectiva actual del pasado.

Los medios que hacen posible esa reconstrucción del pasado, además de la información psicológica y social que tenemos a nuestro alcance, son los vestigios materiales, los ritos, los textos y las tradiciones, pues "cada memoria colectiva requiere del apoyo de un grupo delimitado en el tiempo y el espacio" (Halbwachs, 1992: 84). Es decir, la memoria colectiva actúa como un poderoso agente en la construcción y transformación identitaria de las 
comunidades, pues no se le contempla como si fuera una imagen fija del pasado o una narración absoluta de la historia, ni siquiera como la suma de las memorias individuales, sino que es todo un conjunto de factores que emergen al interior de las comunidades, en sus interacciones dinámicas cotidianas.

Trayendo las ideas de Durkheim y Halbwachs al contexto actual, podemos observar que fue hasta la década de los ochentas cuando las ciencias sociales y las humanidades se interesaron con más brío en el tema de la memoria colectiva como un medio para comprender y reflexionar acerca de los fenómenos actuales, pues, como dice la socióloga inglesa de la memoria Barbara Misztal, ésta "contribuye a nuestra auto-conciencia y nos permite evaluar nuestras potencialidades y límites" (2005: 1328) como individuos y como sociedad. Cabe recordar que, a pesar de que durante gran parte de la modernidad se caracterizó por su amnesia, en la época posmoderna el sujeto más que nunca está obsesionado por reflexionar acerca de su pasado con el fin de encontrar una explicación convincente de las condiciones en que vive en la actualidad y poder mirar con seguridad hacia el futuro cercano, debido a que -como menciona Robert Neelly Bellah (1985), sociólogo norteamericano- las comunidades de la memoria no únicamente están vinculadas con el pasado a través de las reminiscencias, sino que también miran hacia el futuro como comunidades de esperanza, pues no sólo se comparten recuerdos, también aspiraciones e ilusiones.

Al respecto, autores como el historiador norteamericano Jay Winter y su colega francés Pierre Nora advierten que ahora mismo en la escena académica se observa un boom de la memoria (Winter, 2000) que se caracteriza por una "pasión reciente [y creciente] por la memoria" (Nora, 1996: 3), a tal grado que se ha desarrollado una tendencia inter y transdisciplinaria en la que convergen historiadores, sociólogos, humanistas, antropólogos, psicólogos sociales, filósofos, al igual que especialistas de la comunicación masiva y los estudios culturales.

Además, fue a partir de esta recuperación de los trabajos de Durkheim y Halbwachs (especialmente del segundo) cuando una buena cantidad de teóricos comenzaron a proponer nuevos términos que permiten tratar este tema como un objeto de estudio vigente y de interés para las nuevas generaciones de investigadores sociales de diversas nacionalidades. Entre otros, los esposos alemanes Jan Assmann (1995, 2006, 2011) y Aleida Assmann (1996, 2008) hablan de una memoria cultural y una comunicativa, el galo Pierre Nora (1989) introdujo el concepto de lugar de la memoria, la británica Barbara A. Misztal (2003) argumenta acerca de una memoria generacional, y la holandesa José van Dijck $(2004,2007)$ explica en qué consiste la memoria mediada por la tecnología digital.

En nuestro caso, afirmamos que la relación entre nostalgia y memoria se da en el terreno cultural, pero además tiene que ver directamente con el cruce de las experiencias personales y 
colectivas, teniendo los medios masivos de comunicación un rol fundamental en este proceso. Sin embargo, aunque parezca lo más adecuado, no es útil el concepto de memoria cultural para los intereses de esta investigación. Para el matrimonio de egiptólogos Assmann, esta última es una memoria a largo plazo, que está distanciada de la vida diaria, pues pertenece al campo de los ritos, los monumentos y las narrativas milenarias; en cambio, la memoria comunicativa o cotidiana hace alusión a los recuerdos experienciales, vivenciados por los mismos sujetos que recuerdan dentro de un ambiente cultural específico, lo cual hace que se le vincule a un horizonte temporal bien delimitado que no se extiende a más de cien años hacia el pasado; además, las emociones juegan un importante papel en este proceso (Assmann, 1995). El amor, la simpatía, el deseo de pertenencia, el odio, la desconfianza y el dolor, todos ellos ayudan a definir nuestros recuerdos y les proveen de algún aspecto contextual. "Sólo las formas de comunicación estimuladas por la emoción traen consigo estructura, perspectiva, relevancia, definición y contexto para la memoria" (Assmann, 2006: 3).

Entonces, la principal diferencia entre las formas sociales de la memoria y las que pertenecen al orden político y cultural es que las primeras son efímeras porque dependen de las experiencias personales y la comunicación interactiva, mientras que las segundas se emplean a largo plazo porque se transmiten perennemente de generación en generación.

En este caso, explica Aleida Assmann, la memoria comunicativa o cotidiana se presenta en dos dimensiones: 1. la interacción con otros individuos, y 2. la interacción con signos y símbolos externos. Los recuerdos autobiográficos, aunque no pueden ser encarnados por otras personas, sí pueden ser compartidos con otros. Estos, "una vez que son verbalizados en forma narrativa o representada por una imagen visual, llegan a ser parte de un sistema simbólico intersubjetivo y dejan de ser una propiedad puramente exclusiva e inalienable" (2008: 50). Finalmente, en este proceso de codificación los recuerdos pueden ser intercambiados, compartidos, corroborados, confirmados, corregidos, cuestionados y apropiados.

No obstante, estos mismos autores consideran que el concepto de memoria colectiva de Halbwachs no ha perdido vigencia. Al respecto, dicen que éste es un término paraguas que alberga cualquier tipo de memoria grupal o social. Todas las instituciones y los grupos sociales, con la asistencia de símbolos, textos, imágenes, ritos, ceremonias, sitios y monumentos, construyen su memoria y, por lo tanto, su identidad. En este sentido, las memorias colectivas también se caracterizan por su mediación; es decir, a diferencia de la historia -que busca reunir, antes que fragmentar-, éstas se basan en la selección y la exclusión de los recuerdos que resultan trascendentes o irrelevantes para los miembros de cada una de las comunidades de la memoria (Bellah, 1985) o comunidades mnemónicas (Zerubavel, 1996, 2003; Coraiola, Suddaby y Foster, 2018), pues cuando sus miembros se sienten ligados simbólica y 
afectivamente a un pasado compartido, entonces se refuerza su sentido de pertenencia a un grupo social.

De acuerdo con el historiador francés Pierre Nora (1989), historia y memoria no son lo mismo, aun cuando tengan un objeto común: el pasado. La primera se basa en la reconstrucción incompleta y problemática de los eventos, mientras que la segunda es el recuerdo vívido del pasado, por lo cual siempre va a estar presente en grupos de personas que experimentaron los sucesos. La historia es un proceso intelectual, secular y analítico, mientras que la naturaleza de la memoria es afectiva, emotiva, abierta y susceptible. La historia es universal, y simultáneamente todos y nadie tiene derecho de propiedad sobre ella; al contrario, la memoria es múltiple y, por lo tanto, pertenece a cada una de las personas, los grupos y las comunidades. En fin, la historia está atada a las continuidades temporales, a las progresiones y relaciones entre las cosas; las raíces de la memoria se encuentran depositadas en espacios, gestos, imágenes y objetos, o sea, en lo que Nora denomina lugares o sitios de la memoria (lieux de mémoire).

Los lugares de la memoria son restos, pero también son repositorios materiales, simbólicos y funcionales de recuerdos. Específicamente, la sociedad posmoderna es archivística, depende en gran medida de la materialidad de los vestigios, de la inmediatez de la grabación y la visibilidad de la imagen. "Ninguna sociedad ha producido archivos tan deliberadamente como la nuestra, no sólo en volumen, no sólo por los nuevos medios de reproducción y preservación, sino también por su estima supersticiosa, por su veneración al resto" (Nora, 1989: 13). De hecho, la cultura popular de nuestros días es sumamente nostálgica y, por este motivo, está muy ligada a los registros mediáticos, tan cercanos a las experiencias y sentimientos de quienes los consumen.

Es más, Jean Baudrillard (1984) argumentó que, en la posmodernidad, ante un nuevo escenario social carente de sentido, al sujeto le fascina jugar con los vestigios que quedan de la realidad destruida, como un intento de redescubrir un cierto placer en la ironía y el juego de las cosas; pero también, según el sociólogo francés, se puede interpretar como una búsqueda, quizás desesperada, de localizar un lugar -hiperreal- donde vivir con lo que queda. Es como estar sobreviviendo en medio de los vestigios del pasado, tratando de encontrarle sentido al nuevo escenario social.

Entre los lugares simbólicos de la memoria se encuentran la industria mediática y toda la producción cultural sónica y audiovisual que en un contexto establecido se vinculó con la vida cotidiana de los sujetos. En este sentido, se puede afirmar que la memoria colectiva y comunicativa también tiene un marcado carácter estético, desde la visión de que el uso de esos sitios aumenta la posibilidad de recordar-juntos (Halbwachs, 1925/2004) y sentir-juntos

\section{Question, Vol. 1, N. 62 , abril-junio 2019. ISSN 1669-6581}


(Maffesoli, 1988/2004, 2014, 2016) en torno a los bienes simbólicos que formaron parte del paisaje mediático (Appadurai, 1990) en un marco social particular.

Aquí cabe, pues, otra concepción del término memoria cultural, propuesto por Mieke Bal (1999), teórica y crítica cultural de origen holandés, quien dice que la memoria puede ser entendida como un fenómeno cultural, así como es individual y social. Por lo tanto, los miembros de un determinado grupo de personas son capaces de reconstruir un pasado densamente cargado de significado a partir de los recuerdos evocados por los objetos simbólicos que aún consideran importantes en sus vidas personales y colectivas. De ahí que Halbwachs (2004) afirmara que la memoria formada socialmente era más que la simple suma de recuerdos individuales. Incluso, como agrega el israelí Eviatar Zerubavel, en su artículo Social memories: Steps to a Sociology of the past (1996), la memoria colectiva de una comunidad mnemónica incluye sólo aquellos recuerdos que son compartidos comúnmente por todos los miembros. Además, implica la integración de los diferentes pasados personales en un pasado común único que todos los miembros de una comunidad específica recuerdan colectivamente; por lo tanto, se pueden identificar manifestaciones individuales o grupales que giren en torno a ese pasado común. La memoria generacional (Misztal, 2003) es un claro ejemplo de este tipo de reconstrucción mnemónica del pasado.

Cada generación, dada su especificidad y unicidad, vive sus propias experiencias. Es decir, a sus miembros no únicamente les une su posición geográfica, temporal y etaria, sino que su identidad grupal también está enlazada al entorno en que se desarrollan. Según Karl Mannheim (1993), el sociólogo clásico alemán, la etapa de formación de la memoria distintiva y el criterio personal de los miembros de una generación se ubica entre la adolescencia tardía y la adultez temprana, lo cual hace que en este mismo proceso ellos mismos se sitúen en circunstancias sociales, políticas y culturales similares posibilitando que sean compartidas la mentalidad y la sensibilidad de cada época en específico. Explica Barbara Misztal que es durante este periodo de vida donde se localiza el momento crítico para la codificación de la memoria; o sea, cuando la memoria es compartida entre los jóvenes se mantienen vivos los recuerdos y "una memoria generacional, resultado de la exposición común de sus miembros a los procesos sociales e intelectuales, depende del ritmo que tiene el cambio social" (2003: 85). Incluso, como comentan Henry L. Roediger III y James V. Wertsch (2008), académicos de la Universidad Washington de San Luis, Missouri, al escribir acerca de la creciente importancia de los estudios sobre la memoria desde la comunicación y los estudios culturales: "los sociólogos están interesados en cómo las experiencias formativas de una generación (usualmente en sus años de adulto joven) comparten su recuento de eventos históricos y cómo se comparten las características sociales y psicológicas de la generación" (p. 15). 
Entonces, si se habla de la memoria colectiva de las generaciones más recientes necesariamente se tiene que considerar su convivencia cotidiana con los medios de comunicación, las industrias culturales y la cultura popular masiva (pop culture), como lo asumieron el sueco Ron Eyerman y el británico-australiano Bryan S. Turner al mencionar las dimensiones culturales de las generaciones contemporáneas en Outline of a Theory of Generations (1998). Según ellos, la posmodernización de la cultura, a través del uso de dispositivos electrónicos, y la globalización de la cultura pop, permite que las generaciones permanezcan en el tiempo y el espacio, pues se amplía la fluidez en las identidades y las memorias generacionales. En otras palabras, el cine, la música popular (la radio), la televisión y el internet integran el sistema cultural a través del cual los sujetos comparten imágenes, sonidos y rituales que son disfrutados y apropiados por los miembros de la generación, construyendo una base emotiva y afectiva que es compartida por todos ellos, la cultura colectiva. Esto sucede así porque, como sostiene la especialista inglesa en cultura popular Tara Brabazon, en su libro sobre la memoria popular de la Generación X (2005), "no podemos separar las representaciones populares, como la música y el cine, de las experiencias de vida. Las canciones que son significativas son la gramática y el forraje de nuestros problemas, placeres, alegría y dolor" (p. 67).

En cuanto a este aspecto, Misztal (2003) asegura que los medios masivos de comunicación constituyen una de las instituciones de la memoria. Incluso, dice, su papel en la construcción de las memorias colectivas hoy es mucho más importante que el desempeñado por los sistemas político, educativo y jurídico, así como los museos.

\footnotetext{
Hoy, la memoria está más distante de las fuentes tradicionales del poder, mientras que cada vez está más formada por los medios masivos (...). La memoria colectiva es definida como la representación del pasado, 1) el pasado compartido por un grupo, y 2) el pasado que es conmemorado colectivamente, que representa y da sustancia a la identidad grupal, sus condiciones actuales y su visión del futuro (p. 25).
}

Como ejemplo de lo anterior, tomemos en consideración lo que John B. Thompson expone en un texto denominado Tradition and self in a mediated world (1996). En este documento, el sociólogo estadounidense afirma que en las sociedades tradicionales el sentido del pasado se generaba a partir de los encuentros cotidianos cara-a-cara y, por consiguiente, de la oralidad. Sin embargo, desde que se comenzaron a desarrollar sistemas más complejos de comunicación a lo largo de la modernidad, las personas y las comunidades organizan sus recuerdos y reconstruyen el pasado con la intervención de algún medio técnico, es decir, a 
través de alguna forma de interacción o cuasi-interacción mediada; entonces, no únicamente las relaciones sociales, sino también la memoria pasó de la esfera netamente privada a la pública. Además, en el mundo actual, comenta Thompson, estos nuevos sistemas hacen posible la transmisión remota -en tiempo y espacio- de información y contenido simbólico por medio de papel, cables eléctricos y ondas electromagnéticas, lo cual permite que las identidades generacionales sean reforzadas a través de una memoria mediática que se mantiene viva aún con el paso de los años.

Esta fuerte necesidad posmoderna de recuperar los recuerdos colectivos depositados en la producción simbólica de los medios masivos puede reencantar el mundo (parafraseando a Weber), al lado de los recuerdos, las emociones y los sentimientos, dado que la condición estética que afecta a las colectividades es ilimitada y dinámica, contrario a lo que tradicionalmente ha sucedido con las memorias oficiales que son provistas por las instituciones. La cohesión social y las identidades sociales necesitan reforzarse por medio de las relaciones afectivas al interior de los grupos, al mismo tiempo que se reestructuran a través de los recuerdos. Incluso, autores como el antropólogo londinense John Davis (1989) advierten que cada generación es libre de rehacer la historia tomando su herencia de la antecesora y puede rebelarse contra ella creando un nuevo ambiente que será sujeto de resistencia para las generaciones posteriores, de tal suerte que los miembros de las comunidades recuerdan y olvidan lo que creen conveniente para su reproducción social.

En concreto, los recuerdos personales son los ejes centrales en la reconstrucción narrativa de la memoria colectiva, pues cada individuo aporta uno o varios fragmentos de esa unidad incompleta, repasando mentalmente lugares y momentos (ambientes), además de objetos materiales e inmateriales que fueron llenados de significado. Estos referentes contextuales, así como los recordatorios, son auxiliares eficaces al momento de la reconstrucción social y cultural del pasado.

El recuerdo necesita no sólo de receptividad mental sino también de un referente ambiental (contextual) que atrapa la atención de uno. Los recordatorios son objetos externos, condiciones y discursos interpersonales que evocan el contra-proceso interno, unificando fragmentos de experiencia (Hall y Kondora, 2005: 1348).

Respecto a las generaciones que han tenido un estrecho acercamiento con la cultura pop y todas sus expresiones artísticas, como sucedió a partir de los sesenta, definitivamente entre sus recordatorios se encuentran los bienes simbólico-mediáticos. Estos, aunque originalmente fueron diseñados durante el período de mayor expansión capitalista con el fin de convertirse en 
el medio idóneo que enriqueciera a los dueños de las industrias del entretenimiento con la total subvención de las modas, con el paso del tiempo están siendo re-cargados de sentido emotivo por parte de sus mismos consumidores originales, es decir, de quienes los disfrutaron en el momento de ser promovidos por la moda y los medios masivos. Su recuperación mental no sólo sirve para la evocación insustancial y cursi de una representación del pasado, sino también para reconocer una variedad de eventos que resultan importantes e, incluso, sagrados, para quienes comparten alguna historia que se cuenta, con alguna canción, película o serie como telón de fondo. Sucede de esta manera porque se recuerda en respuesta a las necesidades e historias de otras personas; además, porque cuando una memoria es compartida, ésta integra y valora las diversas perspectivas de quienes recuerdan un mismo episodio. Así, la memoria cultural y las narraciones de vida estetizan el mundo cotidiano de los sujetos, al mismo tiempo que dotan de una cierta identidad grupal a su respectiva generación. No obstante, los artefactos simbólicos, ya sean visuales, textuales, sonoros o multimedia, y las tecnologías mediáticas no únicamente intervienen en la mediación de los recuerdos que se tienen del pasado personal, sino también de las relaciones interpersonales y grupales. Consideremos, por tanto, que todas las micro-historias mediadas por las tecnologías culturales y de entretenimiento son el principal recurso socio biográfico con el cual se generan e identifican las comunidades mnemónicas (Zerubavel, 1996, 2003) posmodernas, definiendo qué y a quién recordar, y cuándo debe ser recordado. Como afirma la Dra. José van Dijck, investigadora del área de cultura y comunicación de la Universidad de Amsterdam, las memorias mediadas $(2004,2007)$ integran la autobiografía de cada sujeto, pero también forman una parte importante en la conformación de las identidades culturales de las generaciones contemporáneas. Es más, tanto los medios como los objetos culturales no deben considerarse sólo instrumentos que permiten mantener versiones estáticas del pasado, sino también configuran un espacio simbólico donde culturalmente se encuentran, interactúan y chocan las memorias personales y las colectivas en torno a un pasado que no sólo es compartido sino también recordado en conjunto, o sea, literalmente el pasado es conmemorado.

Para esta académica holandesa, el hablar de memorias mediadas implica que dentro de una metafórica caja de zapatos el nostálgico posmoderno colecciona dos tipos de objetos, saturados ambos de una fuerte carga emocional: por una parte, los documentos que los sujetos mismos han producido como forma de obtener registro de los momentos más significativos de su vida personal (fotos del grupo de amigos, videos familiares, demos musicales captados con la grabadora casera, etcétera) y, por otro lado, objetos mediáticos (discos, cintas, CDs y memorias USB con la música predilecta, videocasetes con grabaciones de los programas 
preferidos y DVDs de las películas favoritas). Sin embargo, estas colecciones individuales de bienes mediáticos no responden simplemente a la moda y al gusto de las generaciones, sino que también pertenecen al terreno de la lucha simbólica, pues al grabar, borrar o regrabar música, las personas se apropian exclusivamente de la parte de la cultura popular con la que se identifican. Entonces, de acuerdo con van Dijck, la tarea de las memorias mediadas no se limita únicamente a la documentación del pasado, sino que también comunican qué y cómo sucedieron las cosas en el entorno social más inmediato, así como en el más amplio.

Particularmente, la música, las películas, los programas televisivos, los diarios y las revistas, a pesar de ser objetos que normalmente son consumidos en forma privada, se han convertido en la interfaz que conecta las vivencias y los sentimientos personales con las experiencias y las emociones compartidas de manera grupal. $\mathrm{Y}$ es en esta relación donde convergen lo psicológico y lo sociológico con los artefactos materiales y simbólicos, las tecnologías y las prácticas culturales, a tal grado que hoy en día se puede afirmar la existencia de toda una industria cultural de la nostalgia.

\section{Bibliografía}

Appadurai, A. (1990). Disjuncture and difference in the global cultural economy. Publicculture, 2(2), 1-24.

Assmann, A. (1996). Texts, traces, trash: The changing media of cultural memory. Representations, 56, 123-134.

Assmann, A. (2008). Transformations between History and memory. Social research, 75(1), 4972.

Assmann, J. (1995). Collective memory and cultural identity. New German critique, 65, 125-133.

Assmann, J. (2006). Introduction: What is "Cultural memory"? En Religionand cultural memory: Ten studies (pp. 1-30). Standford, EUA: Standford University Press.

Assmann, J. (2011). Communicative and cultural memory. En Meusburger, P.; Heffernan, M. y Wunder, E. (Eds.). Cultural memories. The Geographical point of view (pp. 15-28). Dordrecht, Holanda: Springer.

Bal, M. (1999). Introduction. En Bal, M.; Crew, J. y Swpitzer, L. (eds.). Acts of memory. Cultural recall in the present (pp. vii-xvii). Hanover, EUA: University Press of New England.

Baudrillard, J. (1984). Interview: Game with vestiges. On the beach, 5, 19-25. 
Bellah, R. N.; Madsen, R.; Sullivan, W. M.; Swidler, A. y Tipton, S. M. (1985). Habits of the heart: Individualism and commitment in American life. Berkeley, EUA: University of California Press.

Brabazon, T. (2005). From revolution to revelation: Generation X, popular memory and cultural studies. Aldershot, Inglaterra: Ashgate.

Coraiola, D.; Suddaby, R. y Foster, W. M. (2018). Organizational fields as mnemonic communities.

En Glückler, J.; Suddaby, R. y Lenz, R. (Eds.). Knowledge and Institutions (pp. 45-68). Dordrecht, Holanda: Springer.

Davis, J. (1989). The social relations of the production of history. En Tonkin, E.; McDonald, M. y Chapman, M. (Eds.), History and ethnicity (pp. 104-120). Londres, Inglaterra: Routledge.

De la Peza, C. (2001). El bolero y la educación sentimental en México. México, D.F.: Universidad Autónoma Metropolitana-Miguel Ángel Porrúa.

Durkheim, E. (2003). Las formas elementales de la vida religiosa. Madrid, España: Alianza Editorial.

Eyerman, R. y Turner, B. S. (1998). Outline of a Theory of Generations. European journal of social theory, 1(1), 91-106.

Hall, J. M. y Kondora, L. L. (2005). "True" and "false" child sexual abuse memories and Casey's phenomenological view of remembering. American behavioral scientist, 48(10), 13391359.

Halbwachs, M. (2004). Los marcos sociales de la memoria. Barcelona, España: Anthropos.

Halbwachs, M. (1992). On collective memory. Chicago, EUA: The University of Chicago Press.

Maffesoli, M. (2004). El tiempo de las tribus. El ocaso del individualismo en las sociedades modernas. México, D.F.: Siglo XXI.

Maffesoli, M. (2014). El regresar del tiempo. Formas elementales de la posmodernidad. México: Siglo XXI.

Maffesoli, M. (2016). From society to tribal communities. The Sociological review, 64, 739-747.

Mannheim, K. (1993). El problema de las generaciones. Revista española de investigaciones sociológicas, 62, 193-242.

Misztal, B. (2003). Theories of social remembering. Maidenhead, Inglaterra: Open University Press.

Misztal, B. (2005). Memory and democracy. American behavioral scientist, 48(10), 1320-1338.

Nora, P. (1989). Between memory and history: Les lieux de memoire. Representations, 26, 724 
Nora, P. (1996-1998). Realms of memory: Rethinking the French past (3 vols.). Nueva York, EUA:

Columbia University Press.

Roediger III, H. L. y Wertsch, J. V. (2008). Creating a new discipline ofmemorystudies. Memorystudies, 1(1), 9-22.

Thompson, J. B. (1996). Tradition and self in a mediated world. En Heelas, P.; Lash, S. y Morris, P. M. Detraditionalization (pp. 89-108). Oxford, Inglaterra: Blackwell.

van Dijck, J. (2004). Mediated memories: Personal cultural memory as an object of cultural analysis. Continuum: Journal of media and cultural studies, 18(2), 261-277.

van Dijck, J. (2007). Mediated memories in the digital age. Standford, EUA: Standford University Press.

Winter, J. (2000). The generation of memory: Reflections on the memory boom in contemporary historical studies. Bulletin of the German Historical Institute, 27, 69-92.

Zerubavel, E. (1996). Social memories: Steps to a Sociology of the past. Qualitative Sociology, 19(3), 283-299.

Zerubavel, E. (2003). Time maps. Collective memory and the social shape of past. Chicago, EUA: The University of Chicago Press. 